\title{
Zn:MgO:PPLN Waveguides for Rb cold Atom Trap based Quantum Gravitometry in a CubeSat
}

\author{
Lewis G. Carpenter'1, Sam A. Berry' ${ }^{1}$, Thomas H. Legg' ${ }^{2}$, Mark C. Farries², Chloe J. Watson' ${ }^{1}$, \\ Rex H.S. Bannerman ${ }^{1}$, Alan C. Gray ${ }^{1}$, Christopher Holmes ${ }^{1}$, James C. Gates ${ }^{1}$, Peter G.R. Smith ${ }^{1 \& 3}$, \\ and Corin B.E. Gawith ${ }^{1 \& 3}$ \\ 1. Optoelectronics Research Centre, University of Southampton, Southampton, Hampshire, United Kingdom \\ 2. Gooch and Housego, Broomhill Way, Torquay, Devon, United Kingdom \\ 3. Covesion Ltd, Premier Way, Romsey, Hampshire, United Kingdom
}

Satellite based experiments require laser systems that are compact and efficient, which are robust against vibration, radiation and thermal cycling. Supported by Innovate UK's Quantum Technology 'Cold Atom Space PAyload (CASPA)' project we have fabricated PPLN (Periodically Poled Lithium Niobate) ridge waveguides to act as efficient frequency converters for rubidium atom cooling via second harmonic generation (SHG) of telecoms lasers. CASPA is a technology demonstrator for magneto-optical cooling within a micro CubeSat laying the foundations for novel gravitometry and global positioning techniques [1].

With respect to the particular environment of operation we have developed single mode ridge waveguides with metal indiffusion and dicing. We fabricate PPLN ridge waveguides by firstly high-voltage poling of z-cut magnesium doped lithium niobate, then deposit and thermally indiffuse $\mathrm{Zn}$ to create planar optical confinement, followed by ultra-precision ductile dicing to define ridge waveguides and coupling facets. Fig. 1 shows a typical phase matching spectra, pump mode and PPLN waveguide housed in a space rated package. Waveguide packaging is being developed by Gooch \& Housego (UK).

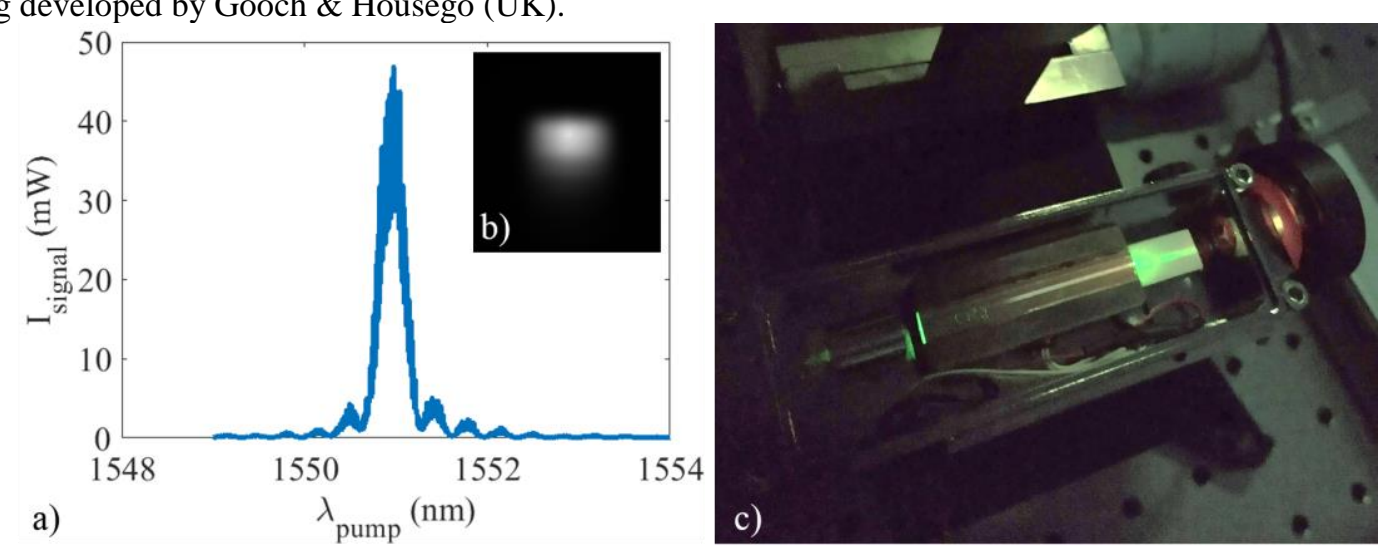

Fig. 1 a) spectra of SHG. b) pump mode of the ridge waveguide. c) image of packaged ridge waveguide notice the build up of the second harmonic (red) and SFG (green) along the waveguide's length.

Fig. 1 a) shows phase matching spectra for a ridge at room temperature for a non-AR coated waveguide; notice the expected $\operatorname{sinc}^{2}$ profile for a uniform PPLN grating. Our non-AR coated waveguides have produced optical to optical efficiencies of up to $22.0 \%$, producing $44.7 \mathrm{~mW}$ of SHG with $203 \mathrm{~mW}$ of transmitted pump. Fig. $1 \mathrm{~b}$ ) shows the pump mode of a waveguide, taken by injecting an EDFA and collecting the light on an AlGaAs CCD beam profiler. The 2nd moment MFD was calculated to be 10.8 and $10.0 \mu \mathrm{m}$ in the $\mathrm{x}$ and $\mathrm{y}$ axes, respectively, allowing efficient mode matching to standard telecommunication fibre. With a free space launch we have measured an average insertion loss, over multiple dies, of $-1.3 \mathrm{~dB}$, for $4 \mathrm{~cm}$ long waveguides that are non-AR coated. When comparing to other ridge PPLN waveguides this is a $3.7 \mathrm{~dB}$ improvement over competing examples of a $5 \mathrm{~cm}$ bonded and thinned device [2] and a $1.9 \mathrm{~dB}$ improvement for a $1 \mathrm{~cm}$ proton exchange device [3]. These low insertion losses can be attributed to both the symmetric pump mode of the waveguides enabling efficient mode matching and low amounts of optical scattering from waveguide sidewall roughness. We have shown ductile dicing to produce an average surface roughness of $0.29 \mathrm{~nm}$ ( $\mathrm{Sa}$ ) on waveguide facets in a single processing step [4].

We will present our latest work on ruggedised $\mathrm{Zn}: \mathrm{MgO}: \mathrm{PPLN}$ ridge waveguides and report on fabrication, insertion/waveguide loss, second harmonic generation spectra/efficiency, vibration and radiation testing of our waveguides for operation in space, and their use in the CASPA project.

\section{References}

[1] T. Fryer, "Cold atoms at space probe's heart," Engineering and Technology, 13, 1 (2018)

[2] T. Umeki, O. Tadanaga, and M. Asobe, "Highly efficient wavelength converter using direct-bonded PPZnLN ridge waveguide," IEEE J. Quantum Electron, 46, 1206-1213 (2010).

[3] J. Sun and C. Xu, "466 mW green light generation using annealed proton-exchanged periodically poled $\mathrm{MgO}^{\mathrm{LiNbO}}$ ridge waveguides,” Opt. Lett., 37, 2028-2030 (2012).

[4] L.G. Carpenter, S.A. Berry, and C.B.E. Gawith "Ductile dicing of $\mathrm{LiNbO}_{3}$ ridge waveguide facets to achieve $0.29 \mathrm{~nm}$ surface roughness in single process step," Electron. Lett., 53, 1672 - 1674 (2017). 